\title{
Ancestry and frequency of genetic variants in the general population are confounders in the characterization of germline variants linked to cancer
}

Anna Bobyn ${ }^{1,2,3+}$, Mehdi Zarrei ${ }^{4,5,6+}$, Yuankun Zhu' ${ }^{7}$, Mary Hoffman ${ }^{1,2,8}$, Darren Brenner ${ }^{9}$, Adam C. Resnick ${ }^{7}$, Stephen W. Scherer ${ }^{4,5,6^{*}}$ and Marco Gallo ${ }^{1,2,8^{*}}$ (D)

\begin{abstract}
Background: Pediatric high-grade gliomas (pHGGs) are incurable malignant brain cancers. Clear somatic genetic drivers are difficult to identify in the majority of cases. We hypothesized that this may be due to the existence of germline variants that influence tumor etiology and/or progression and are filtered out using traditional pipelines for somatic mutation calling.

Methods: In this study, we analyzed whole-genome sequencing (WGS) datasets of matched germlines and tumor tissues to identify recurrent germline variants in PHGG patients.

Results: We identified two structural variants that were highly recurrent in a discovery cohort of 8 pHGG patients. One was a $\sim 40 \mathrm{~kb}$ deletion immediately upstream of the NEGR1 locus and predicted to remove the promoter region of this gene. This copy number variant (CNV) was present in all patients in our discovery cohort $(n=8)$ and in $86.3 \%$ of patients in our validation cohort ( $n=73$ cases). We also identified a second recurrent deletion $55.7 \mathrm{~kb}$ in size affecting the BTNL3 and BTNL8 loci. This BTNL3-8 deletion was observed in 62.5\% patients in our discovery cohort, and in $17.8 \%$ of the patients in the validation cohort. Our single-cell RNA sequencing (scRNA-seq) data showed that both deletions result in disruption of transcription of the affected genes. However, analysis of genomic information from multiple non-cancer cohorts showed that both the NEGR1 promoter deletion and the BTNL3-8 deletion were CNVs occurring at high frequencies in the general population. Intriguingly, the upstream NEGR1 CNV deletion was homozygous in $~ 40 \%$ of individuals in the non-cancer population. This finding was immediately relevant because the affected genes have important physiological functions, and our analyses showed that NEGR1 expression levels have prognostic value for pHGG patient survival. We also found that these deletions occurred at different frequencies among different ethnic groups.

(Continued on next page)
\end{abstract}

\footnotetext{
* Correspondence: stephen.scherer@sickkids.ca; marco.gallo@ucalgary.ca

${ }^{\dagger}$ Anna Bobyn and Mehdi Zarrei contributed equally to this work.

${ }^{4}$ The Centre for Applied Genomics and Program in Genetics and Genome Biology, The Hospital for Sick Children Research Institute, Toronto, Ontario, Canada

${ }^{1}$ Charbonneau Cancer Institute, Cumming School of Medicine, University of Calgary, Calgary, Alberta, Canada

Full list of author information is available at the end of the article
}

(c) The Author(s). 2020 Open Access This article is licensed under a Creative Commons Attribution 4.0 International License, which permits use, sharing, adaptation, distribution and reproduction in any medium or format, as long as you give appropriate credit to the original author(s) and the source, provide a link to the Creative Commons licence, and indicate if changes were made. The images or other third party material in this article are included in the article's Creative Commons licence, unless indicated otherwise in a credit line to the material. If material is not included in the article's Creative Commons licence and your intended use is not permitted by statutory regulation or exceeds the permitted use, you will need to obtain permission directly from the copyright holder. To view a copy of this licence, visit http://creativecommons.org/licenses/by/4.0/. The Creative Commons Public Domain Dedication waiver (http://creativecommons.org/publicdomain/zero/1.0/) applies to the data made available in this article, unless otherwise stated in a credit line to the data. 
(Continued from previous page)

Conclusions: Our study highlights the need to integrate cancer genomic analyses and genomic data from large control populations. Failure to do so may lead to spurious association of genes with cancer etiology. Importantly, our results showcase the need for careful evaluation of differences in the frequency of genetic variants among different ethnic groups.

Keywords: Whole-genome sequencing, Single-cell RNA-seq, Pediatric high-grade glioma, Germline variants

\section{Background}

Brain cancers have recently surpassed leukemias as the number one killer in the pediatric cancer patient population [1]. This appears largely attributable to significant improvements in the clinical management of some leukemia subtypes, whereas no significant progress has been registered for the malignant brain cancer population.

Pediatric high-grade gliomas (pHGGs; World Health Organization grade III and IV tumors), including glioblastoma (GBM), have particularly dismal prognoses [2]. Current treatments usually include maximal safe resection of the main tumor mass followed by local radiotherapy. Some pHGG patients also receive chemotherapy, although this treatment is not uniform and varies depending on the specific patient, prescribing oncologist and treating centre. Temozolomide, which has shown some efficacy in prolonging overall survival in adult GBM patients, is sometimes administered to pHGG patients as well, although clinical trials failed to show efficacy for this drug in pediatric cohorts $[3,4]$. New treatment options are therefore needed to tackle these universally lethal malignancies.

Several genomic studies have shown that pHGGs have low mutational burdens, similarly to other childhood cancers [58]. The mutational landscape of pHGGs is very different from their adult counterparts. For instance, our analyses using cBioPortal and pedcBioPortal show that whereas EGFR is mutated in 53\% of adult GBM samples, and PTEN is altered in $31 \%$ of cases $(n=281$ samples described in reference [9]), these genes are mutated in 6 and 4\% of pHGG cases, respectively $(n=1257$ cases described in references $[10,11])$. Highly recurrent mutations in pHGGs include mutations of genes encoding the histone 3 variant $\mathrm{H} 3.3$, including $21 \%$ of cases with mutations in the $H 3 F 3 A$ gene. H3.3 mutations tend to co-occur with TP53 and ATRX mutations, and are very rare in adult HGGs $[6,12,13]$.

Molecular studies and work with genetic mouse models have shown that co-occurrence of H3.3 and Tp53 mutations cooperate with either overexpression of Pdgfra or loss of Nf1 to drive cancer initiation and progression [14, 15]. However, the majority of human pHGG cases lack these concurrent mutations and their genetic drivers are difficult to infer.

We have recently reported a whole-genome sequencing (WGS) analysis of a collection of pHGGs [5]. In that study, we showed that pHGGs are genomically complex cancers that harbor multiple coexisting genetic subclones. Among the truncal mutations (ie variants that are shared by virtually all the subclones detected in a tumor), we found no obvious candidate driver events in most tumors, except for the above-mentioned H3.3/ TP53/ATRX axis.

Traditionally, somatic mutations are called by comparing WGS data for the tumor tissue and germline (usually peripheral blood) to subtract variants that are specific to the individual patient. The underlying assumption of this method is that germline variants are not informative for cancer etiology. However, recent publications have shown that about 7-8\% of pediatric cancer patients harbor deleterious mutations in at least one of 149 genes with known association to cancer [16]. This frequency might be an underestimation because more than these 149 genes might drive specific cancer types. We hypothesized that the lack of clear genetic drivers in the majority of pHGGs might be an artifact due to the removal of informative germline events that could predispose an individual to the development of the malignancy and/or affect disease progression. Therefore, we analyzed germline and tumor WGS data separately, and then looked specifically for structural variants that were shared between the germline and the tumor tissue and that recurred in multiple pHGG patients. Our analyses identified two structural variants that were highly recurrent in the pHGG population. However, subsequent analyses with datasets derived from a control population of thousands of individuals revealed that these variants are present at high frequency in the non-cancer population. Of interest, we found that these variants occurred with different frequencies in different ethnic groups.

Our findings highlight the need to contextualize findings from cancer genomic studies with genomics data from noncancer cohorts in order to properly identify putative cancer predisposing genes. This is especially relevant now that significant efforts are being invested in uncovering predisposing germline variants for different cancer types, including adult malignancies. This task will be increasingly enabled by efforts from large consortia that are collecting genomic information from the general population.

\section{Methods pHGG samples}

Samples used for WGS with linked reads for the pHGG discovery cohort ( $n=8$ patients) were recently described in Hoffman et al. [5]. 


\section{Non-cancer control cohort}

The large non-cancer control cohort comprises of 2596 genome sequences hosted at the Centre for Applied Genomics at the Hospital for Sick Children, Toronto [17]. They are parents and unaffected siblings of individuals from our disease sequencing studies. We also analyzed other population control data from Personal Genome Project Canada (PGPC; $n=93$ ) [18] and 1000 Genomes Project CNVs obtained from the Database of Genomic Variants (DGV; $n=2504)$ [19].

\section{Visualization of genomic data}

Data generated by WGS with linked reads were visualized with Loupe version 2.1.2 (10xGenomics). scRNAseq data were visualized with Loupe Cell Browser version 3.0.1 (10xGenomics). The single-cell transcriptomics data for human hippocampus and cortex [20] was accessed and analyzed through the Single Cell Portal (https://singlecell.broadinstitute.org/single_cell), a web interface hosted by the Broad Institute.

\section{Survival plots}

Survival analysis was done using a previously published pHGG cohort [21] with the R2 Genomic Analysis Visualization Platform (https://hgserver1.amc.nl/cgi-bin/ r2/main.cgi). Patients were stratified based on NEGR1 expression, with NEGR1-low cases corresponding to the bottom quartile of expression. Statistical analysis was performed with the log-rank test.

\section{Graphing software}

Pie charts and histograms were generated with Prism 8 (GraphPad).

\section{Results}

Identification of recurrent genetic variants at the NEGR1 locus in pHGGs

We have profiled pHGG samples by WGS with linkedread technology (10xGenomics), as recently described [5]. Linked-reads allow the reconstruction of long (Mbp) haplotypes at the level of individual chromosomes and are optimal for the identification and visualization of

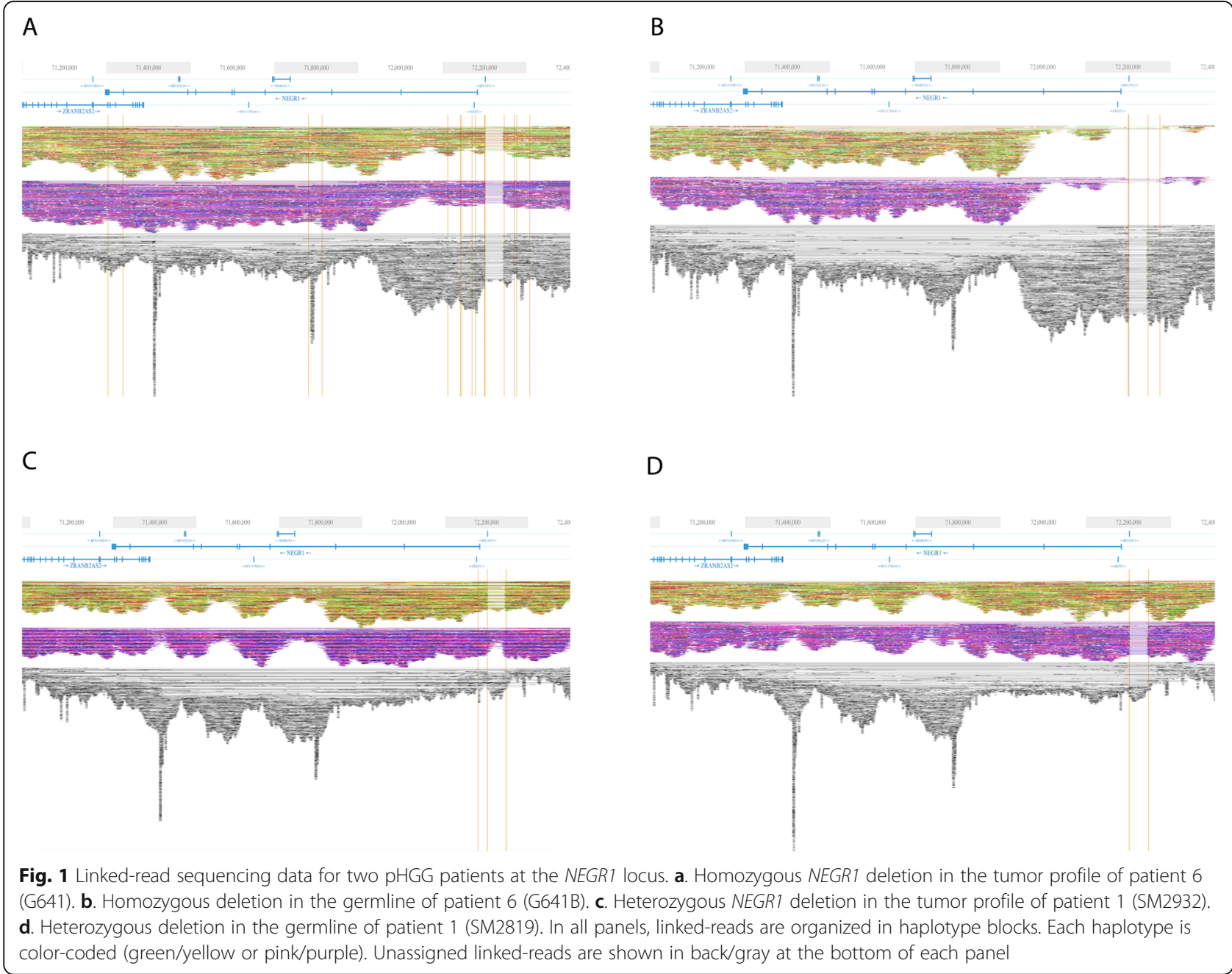


structural variants. In particular, because maternal and paternal haplotypes are determined by analysis of singlenucleotide polymorphisms along the chromosome length, this experimental set up allows assignment of structural variants to a specific haplotype. We generated WGS datasets for matched tumor tissue and blood samples (germline controls) from a discovery cohort of 8 pHGG patients. In addition, relapse samples were available for 4 patients, including 3 relapses for one patient (patient samples and anagraphical information were described in ref. [5] and are summarized in Supplemental Table S1). We were surprised to observe $100 \%$ of our tumor samples displaying a deletion immediately upstream of the Neuronal Growth Regulator 1 (NEGR1) gene. Five out of eight had homozygous deletions (see Fig. 1a-b for examples), whereas three out of eight pHGG patients from our cohort harboured a heterozygous deletion in the NEGR1 region (Fig. 1c-d; Table 1). The deletions were found both in the germline and in tumor tissue. The NEGR1 protein is a member of the IgLON subgroup of the immunoglobin superfamily and has been shown to contain a GPI-anchor attachment site that localizes to lipid rafts [22] and is involved in the maturation and remodelling of the central nervous system [23]. Knockout of the Negr1 gene in mouse models results in defective neuronal maturation [24]. The cell adhesion molecule encoded by NEGR1 has also been reported to be down-regulated in many human cancers. In ovarian cancer, NEGR1 has been proposed as a tumour suppressor gene [22]. Additionally, low NEGR1 expression is correlated with a low survival probability in neuroblastoma, according to a previous study [25]. Given the role of NEGR1 in neural development and cancer progression, this gene proves to be an intriguing subject in brain cancer research. Additionally, our data raised the possibility that mutations in this gene may be germline-predisposing events and deserved follow up studies.

Table 1 Summary of the frequencies of NEGR1 and BTNL8BTNL3 deletion

\begin{tabular}{lll}
\hline & $\begin{array}{l}\text { NEGR1 } \\
\text { deletion }\end{array}$ & $\begin{array}{l}\text { BTNL8-BTNL3 } \\
\text { deletion }\end{array}$ \\
\hline Calgary Cohort $(n=8)$ & $100 \%$ & $62.5 \%$ \\
CBTTC $(n=73)$ & $86.3 \%$ & $17.8 \%$ \\
MSSNG controls $(n=2596)$ & $87.1 \%$ & $48.0 \%$ \\
1000 Genomes Project $(n=2504)$ & $89 \%$ & $38.2 \%$ \\
$\begin{array}{l}\text { Personal Genome Project Canada } \\
(n=93)\end{array}$ & $77.4 \%$ & $48.4 \%$ \\
\hline
\end{tabular}

Datasets include the Calgary cohort, a pediatric HGG dataset from the CBTTC and individuals from the general population (coded parental control Canadian samples in MSSNG); 1000 Genomes Project CNVs obtained from the Database of Genomic Variants [DGV]; and control samples from Personal Genome Project Canada (PGPC)). Deletions are either heterozygous or homozygous
Datasets include the Calgary cohort, a pediatric HGG dataset from the CBTTC and individuals from the general population (coded parental control Canadian samples in MSSNG); 1000 Genomes Project CNVs obtained from the Database of Genomic Variants [DGV]; and control samples from Personal Genome Project Canada (PGPC)). Deletions are either heterozygous or homozygous.

\section{Low NEGR1 expression is associated with worse prognosis in pHGGs}

Because the deletion we observed at the NEGR1 locus was immediately upstream of the gene, we predicted that this lesion might affect the ability of the promoter region to properly activate transcription. Analysis of ENCODE data for histone marks linked to active promoter and enhancer elements supported the notion that the deletion might remove regions that are important for NEGR1 transcription (Supplemental Figure S1).

To further assess the possibility that the deletion upstream of NEGR1 might affect the expression of this gene in pHGG, we re-analyzed single-cell RNA-seq data that our group recently generated from two patientderived xenografts [5] and looked specifically at expression of NEGR1 in these samples. Both xenografts were derived from samples in the Calgary cohort that were profiled with linked-read WGS and had homozygous deletions upstream of $N E G R 1$. We found that neither xenograft expressed appreciable amounts of NEGR1 (Fig. 2a,b). In contrast, transcription of ZRANB2, a gene immediately downstream of NEGR1, was detected in our scRNA-seq datasets (Supplemental Figure S2A,B). In addition, transcription of NEGR1 was detected in previously published single-cell transcriptomic datasets generated from the adult human brain [20] (Supplemental Figure S2C,D). Overall, these data indicate that deletions of the NEGR1 promoter region in pHGGs may result in abrogation of gene expression in pHGG. However, the role of other factors (including epigenetic mechanisms) in repressing NEGR1 transcription cannot be ruled out at this time.

We also looked at the effects of NEGR1 expression on overall survival in a previously published pHGG patient cohort [21]. We found that low expression of NEGR1 was significantly associated with shorter overall survival in this cohort (Fig. 2c). Overall, our data suggest that genetic events affecting NEGR1 expression might have deleterious effects on the survival of pHGG patients.

Our discovery cohort was composed of 8 pHGG patients, a number that limits predictions of applicability of our findings to the larger patient population. We therefore explored whether the deletions at the NEGR1 locus could be identified in a larger cohort of 73 pHGG patients collected by the Children's Brain Tumour 
A

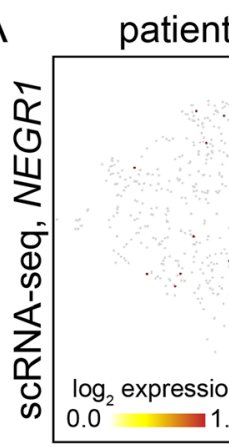

B

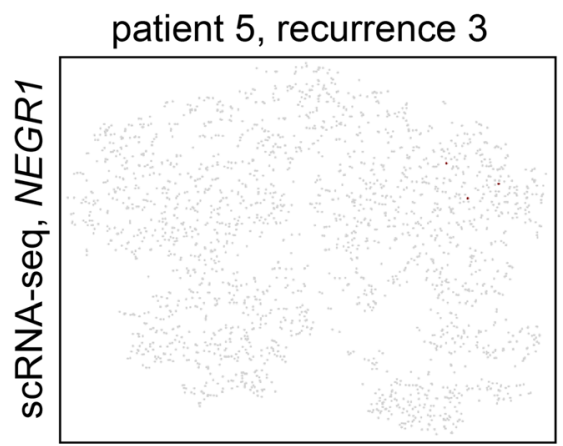

$\mathrm{C} \geq 1.07 \mathrm{~T}$-NEGR1 low $(\mathrm{n}=12)$

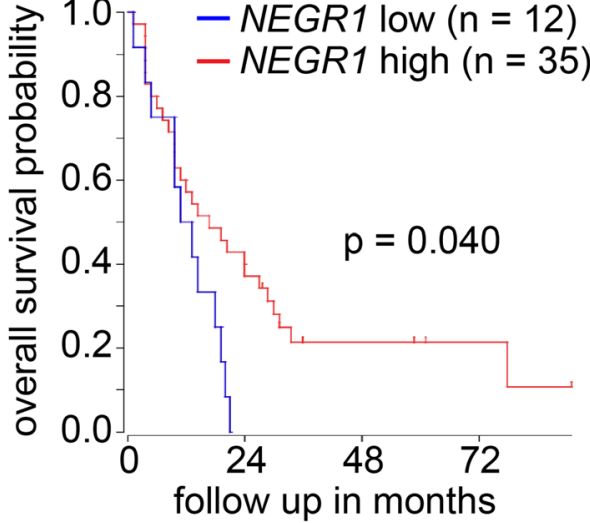

Fig. 2 Single cell RNA-sequencing of NEGR1 expression levels. a. tSNE plot showing single cell RNA-sequencing data illustrates NEGR1 transcription levels in a xenograft derived from recurrence one of patient 3. b. tSNE plot showing NEGR1 transcription levels in single cell RNA-sequencing datasets generated from a xenograft derived from the third recurrence of patient 5. c. A Kaplan-Meier Curve for patient populations with either high or low expression of NEGR1

Tissue Consortium (CBTTC) [26]. We found that the deletion upstream of NEGR1 was present in 63 out of 73 patients (frequency of $86.3 \%$; Table 1).

\section{Recurrent germline deletions at the BTNL3 and BTNL8 loci in pHGG patients}

Intrigued by these findings, we searched for other recurrent germline structural variants in our WGS datasets. We observed frequent deletions $(55.7 \mathrm{~kb})$ in the genomic region encompassing the genes BTNL3 and BTNL8. Overall, this deletion was homozygous in 2 of 8 patients (Fig. 3a, b), and heterozygous in three out of eight pHGG patients (Fig. 3c, d) in our cohort (Table 1). This deletion was also present in patients' germlines (Fig. 3). Butyrophilin (BTN)-like molecules are a part of the B7 family of proteins, which are involved in immune response. The role of the B7 family in regulating the primary immune response against cancer was previously highlighted in clinical trials using monoclonal antibodies against PD-1 and B7-H1 [27, 28]. BTNL8 has two alternatively spliced forms, B7-like and BTN-like. The extracellular domain has been reported to bind the surface of $\mathrm{T}$ cells, co-stimulating proliferation and cytokine production [29]. Although there is little known about the functional role of BTNL3, its downregulation was reported in colon cancer alongside BTNL8 [30]. The frequency of the BTNL3-8 deletion was $17.8 \%$ in the pHGG CBTTC cohort (Table 1). These data confirm that this deletion is frequent in the pHGG population.

\section{Frequency of the NEGR1 and BTNL3-8 deletions in the general population}

The sequence-level breakpoints of the deletions upstream NEGR1 are chr1:72,766,325-72,811,839 (hg19) and were similar among different ethnicities. However, breakpoints of the deletions impacting BTNL8-BTNL3 occurred in repeat regions, thus the exact coordinates were not identifiable due to the complexity of the genomic region. Because the frequency of deletions at the NEGR1 and BTNL3-8 loci was relatively high in pHGG patients, we examined whether these genetic variants were specific to or enriched in the pHGG population. We therefore determined the frequency of these deletions in a large non-cancer cohort that includes genomic information on 2596 individuals [17]. We found that this population control dataset had an NEGR1 deletion frequency of $87.1 \%$ (Table 1; Fig. 4a), comparable to the frequency $(86.3 \%)$ that we observed in the CBTTC pHGG cohort. Our results also show that the BTNL3-8 deletion was detectable in $48.0 \%$ of the controls assessed 


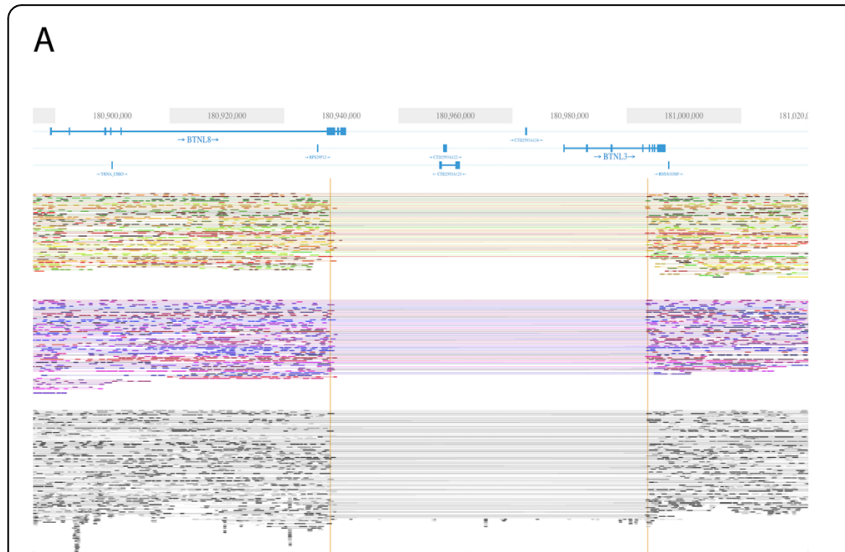

\section{B}

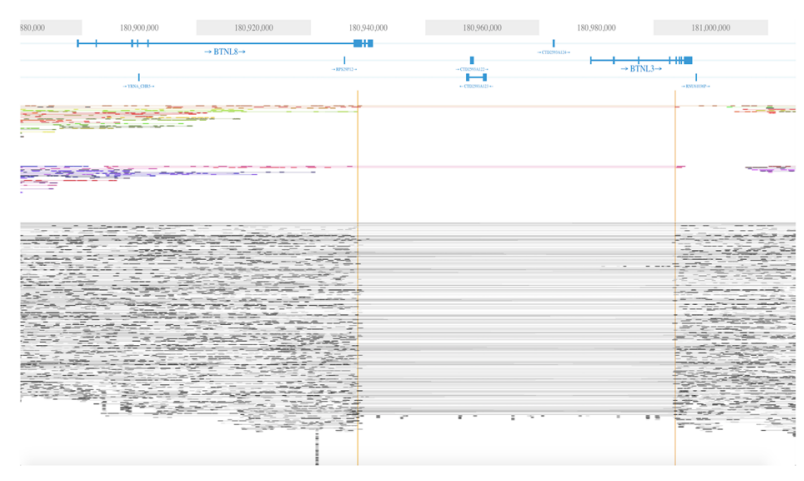

C

D
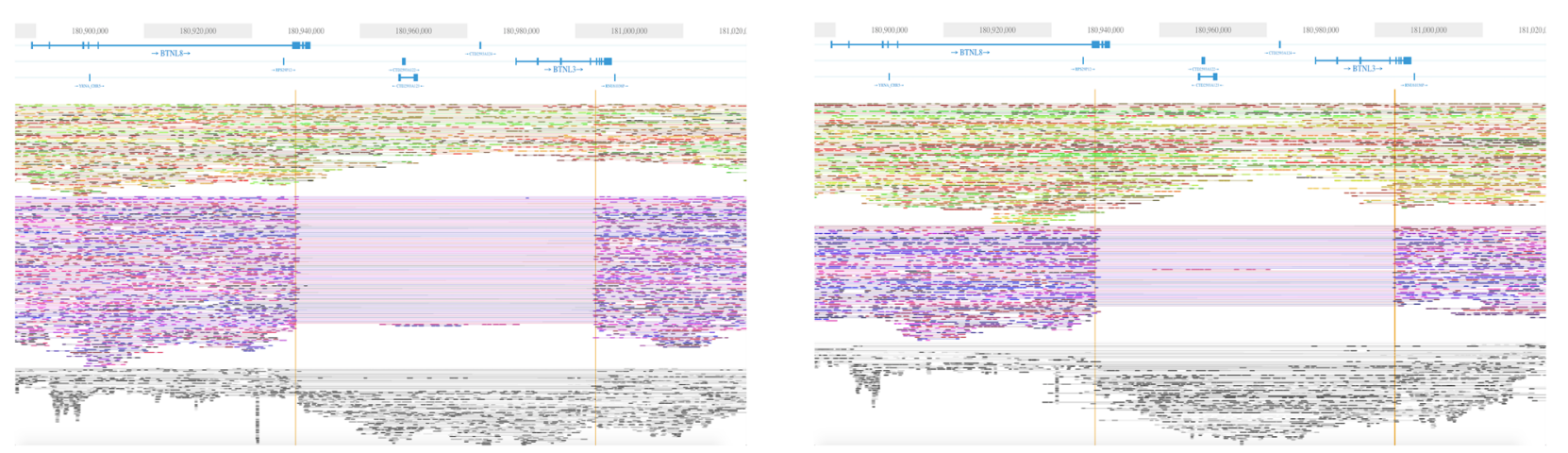

Fig. 3 Linked-read sequencing data for two PHGG patients at the BTNL8-BTNL3 locus. a Homozygous BTNL8-BTNL3 deletion in the tumor profile of patient 6 (G641). b Homozygous deletion in the germline of patient 6 (G641B). c Heterozygous BTNL8-BTNL3 deletion in the tumor profile of patient 1 (SM2932). d Heterozygous deletion in the germline of patient 1(SM2819)

(Table 1; Fig. 4b), higher than the frequency (17.8\%) we observed in the CBTTC pHGG cohort (Table 1). Contrary to our expectations, these data indicate that the NEGR1 and BTNL3-8 germline deletions are relatively common in the general population, and do not appear to be specifically over-represented in the pHGG population. We also analyzed 1000 Genome WGS datasets $(n=$ 2504) with copy number variation (CNV) deposited in the Database of Genomic Variants (DGV) [19, 31], which is the most comprehensive curated public opensource repository for CNVs from population controls. We found a frequency of $89 \%$ for the deletion upstream of NEGR1 and $38.2 \%$ for that impacting BTNL3-8, an observation similar to the earlier control findings.

The frequency of NEGR1 and BTNL3-8 deletions varies in different ethnic groups

Further investigation of the non-cancer population revealed frequency differences of the deletions at the NEGR1 and BTNL3-8 loci between six human populations: European, East Asian, South East Asian, African, American, and "Other". At the NEGR1 locus, the most dramatic difference was observed between the East Asian and African cohorts. The East Asian cohort had the highest frequency of NEGR1 deletions with only $2.5 \%$ of the population having no deletion in comparison to $28.6 \%$ in the African population (Fig. 4c, Table 2).

Frequencies of the control collection $(n=2596)$ stratified by ethnic groups and homozygous or heterozygous deletion types.

Similarly, the East Asian cohort had homozygous and heterozygous deletions of 80.5 and $16.9 \%$ respectively, as opposed to 14.3 and $57.1 \%$ in the African population (Fig. 4c). These cohorts were statistically significant with $p$-value $<0.00001$ by Chi-Square analysis (Table 3 ).

The BTNL3-8 deletion also had different frequencies between ethnic groups (Fig. 4d, Table 2). The largest differences were observed between European and South East Asian descent, with 48.6 and $82.5 \%$ of the respective populations showing no deletion at this locus (Fig. 4d). The European and South East Asian groups were statistically significant with $\mathrm{p}$-value $<0.00001$ by Chi-Square analysis (Table 4). These data therefore illustrate the large variability in frequency of germline genetic variants 


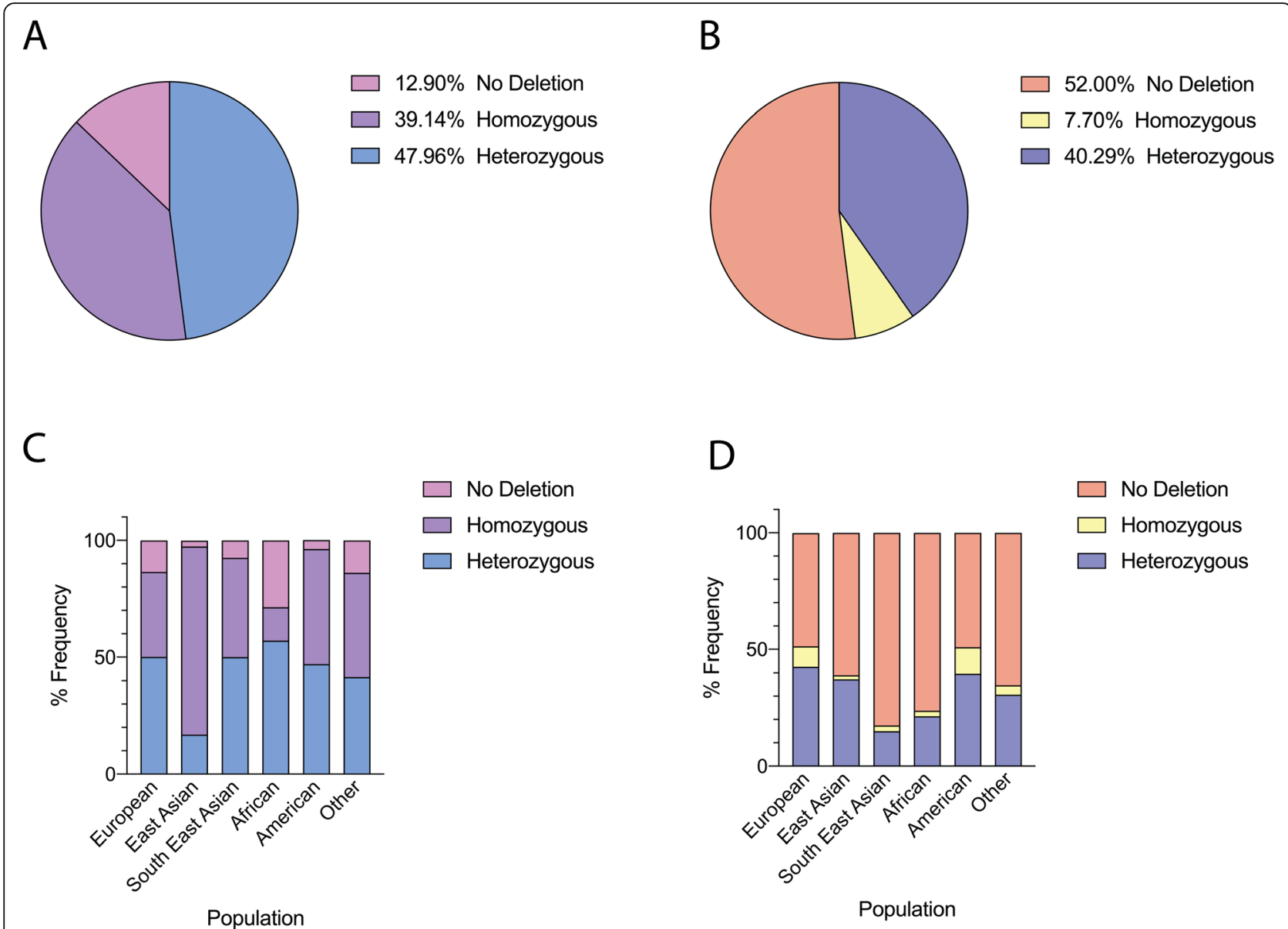

Fig. 4 NEGR1 and BTNL8-BTNL3 deletion frequencies in the general population. a. Frequency of NEGR1 deletions in the general population for all ethnicities. b. Frequency of BTNL8-BTNL3 deletions in the general population for all ethnicities. c. NEGR1 deletions stratified by European, East Asian, South East Asian, African, American, and "Other" descent. d. BTNL8-BTNL3 deletions stratified by European, East Asian, South East Asian, African, American, and "Other" descent

between ethnic groups, a factor that should be incorporated into studies aimed to identify novel germline variants in cancer populations.

\section{Discussion}

The identification of germline genetic variants that might predispose to cancer is an emerging theme in the field of cancer genomics. The identification of such variants holds the promise to incorporate genetic tests as part of early detection strategies for some cancers. Such strategies would be particularly important for pHGG, which is universally lethal. High-profile studies have shown that a significant fraction of the pediatric cancer population carries germline variants in genes known to be cancer drivers or that are associated with cancer etiology and progression [16]. We think it is

Table 2 NEGR1 and BTNL8-BTNL3 deletions in population controls

\begin{tabular}{|c|c|c|c|c|}
\hline & \multicolumn{2}{|l|}{ NEGR1 deletion } & \multicolumn{2}{|c|}{ BTNL8-BTNL3 deletion } \\
\hline & Homozygous & Heterozygous & Homozygous & Heterozygous \\
\hline European $(n=2101)$ & $36.4 \%$ & $50.1 \%$ & $8.6 \%$ & $42.7 \%$ \\
\hline East Asian $(n=118)$ & $80.5 \%$ & $16.9 \%$ & $1.7 \%$ & $37.3 \%$ \\
\hline South East Asian $(n=80)$ & $42.5 \%$ & $50.0 \%$ & $2.5 \%$ & $15.0 \%$ \\
\hline African $(n=42)$ & $14.3 \%$ & $57.1 \%$ & $2.4 \%$ & $21.4 \%$ \\
\hline American $(n=53)$ & $49.1 \%$ & $47.2 \%$ & $11.3 \%$ & $39.6 \%$ \\
\hline Other $(n=202)$ & $44.6 \%$ & $41.6 \%$ & $4.0 \%$ & $30.7 \%$ \\
\hline
\end{tabular}

Frequencies of the control collection $(n=2596)$ stratified by ethnic groups and homozygous or heterozygous deletion types 
Table 3 Chi-square analysis of NEGR1 deletions in the general population

\begin{tabular}{lccccc}
\hline & East Asian & African & American & SE Asian & Other \\
\hline European & 91.8 & 12.8 & 6.03 & 2.88 & 6.00 \\
East Asian & & 62.1 & 18.0 & 30.3 & 40.4 \\
African & & & 18.6 & 15.2 & 14.6 \\
American & & & & 1.09 & 4.13 \\
SE Asian & & & & & 2.90 \\
\hline
\end{tabular}

Analysis of the parental controls from the MSSNG cohort. Values are statistically significant to $95 \%$ above the critical value $5.99(\mathrm{df}=2$ ) and shown in blue. Statistically insignificant results are shown in red

important to stress that most of the evidence to define these variants as "drivers" derived from studies of adult cancers. It is however possible that the mutational dependencies of childhood and adult cancers might be divergent. This case is well exemplified by the radically different incidence of specific genetic alterations in EGFR and $H 3 F 3 A$ in pediatric and adult HGGs, as we mentioned in the introduction to this manuscript. There is therefore promise in efforts to identify new genetic variants that may act as specific drivers of childhood cancers.

Here, we highlight potential confounding factors in the process of identification of new candidate germline variants associated with cancer. Specifically, our work identified two variants affecting genes that were very attractive candidate cancer-predisposing loci, based on their known function and previously published evidence of their involvement in several malignancies. However, these variants were relatively frequent in non-cancer human populations, with marked differences in frequency based on ancestry.

We have identified highly recurrent deletions at two sites - NEGR1 and BTNL3-8 - in the genomes of pHGG patients. From the perspective of a discovery platform, both sites were intriguing because of the biological functions of the genes affected by the lesions. NEGR1 was previously shown to have an important role in neural development [23, 24]. In particular, work with genetic mouse models showed that Negr1 is required for terminal differentiation of neurons and for their ability to

Table 4 Chi-square analysis of BTNL8-BTNL3 deletions in the general population

\begin{tabular}{lcccll}
\hline & East Asian & African & American & SE Asian & Other \\
\hline European & 10.7 & 12.7 & 0.556 & 35.4 & 21.5 \\
East Asian & & 3.53 & 8.21 & 11.7 & 2.42 \\
African & & & 7.82 & 0.799 & 1.87 \\
American & & & & 17.1 & 6.97 \\
SE Asian & & & & & 8.12 \\
\hline
\end{tabular}

Analysis of the parental controls from the MSSNG cohort. Values are statistically significant to $95 \%$ above the critical value $5.99(\mathrm{df}=2)$ and shown in blue. Statistically insignificant results are shown in red properly form synapses. The deletions we identified in pHGG patients are predicted to affect the regulatory regions of the gene. This prediction is supported by our scRNA-seq data, which showed undetectable levels of NEGR1 transcripts in two patient-derived xenograft models. Based on all these data, it would be reasonable to conclude that NEGR1 may play a role in the etiology of pHGG.

However, our analyses of non-cancer populations clearly show that the NEGR1 promoter deletion is present in a majority of individuals in the general population. Based on this finding, it is therefore difficult to support the notion that NEGR1 might be involved in tumor etiology in the context of pHGG, and possibly other cancers as well. We found, however, an association between low expression of NEGR1 and poor overall survival in pHGG patients. It is therefore possible that deletions that negatively affect NEGR1 expression might have modulatory effects on brain tumors and have negative prognostic value. This would be interesting, because it would exemplify that some common germline variants could have effects on tumor progression.

Our finding that the region upstream of NEGR1 is homozygously deleted in $\sim 40 \%$ of individuals in the general population is particularly intriguing. Since mouse models with homozygous Negr1 deletions have neural defects, our data raise the question of whether the murine and human orthologues paly similar roles in brain development. Our data seem to challenge this notion. Another possibility is that the human lineage developed compensatory mechanisms that can overcome loss of NEGR1 expression during neural development, whereas Negr 1 plays a more pivotal role during mouse development.

Recent publications have shown that some cancer patients carry deleterious variants of established cancer genes in their germlines, suggesting that some individuals may be predisposed to developing some malignancies [16, 32]. Cancer initiation and progression may therefore be modulated by the interplay and crosstalk between germline and somatic variants. Our present work highlights the need for comparing the frequencies of putative cancer predisposition variants in the germlines of cancer patients and non-cancer populations. A cancer-centric perspective may result in the identification of germline variants that are relatively frequent in the general population. These comparisons are made easier by large genomic datasets that are being collected by international efforts.

In addition, our data show major differences in the frequencies of the deletions at the NEGR1 and BTNL3-8 loci between different ethnic groups. These results highlight the need to cross-reference the frequencies of germline variants with non-cancer populations with 


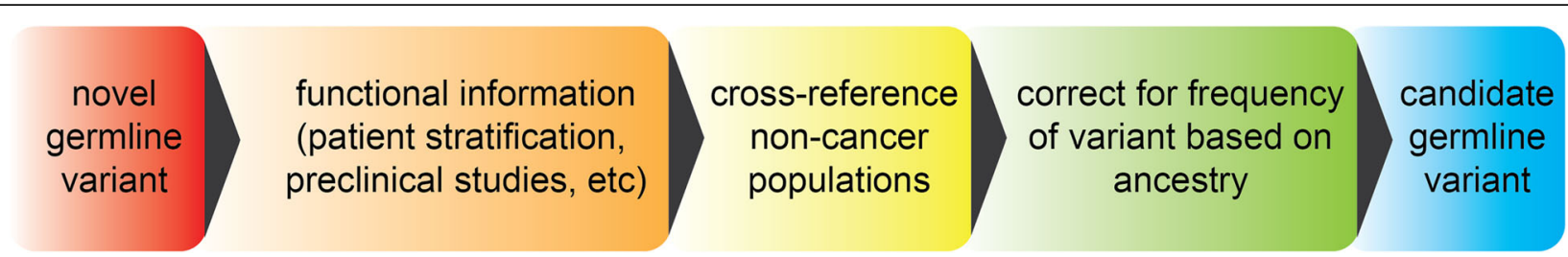

Fig. 5 Model workflow for the identification of novel candidate germline variants associated with cancer. We suggest several filters to identify candidate cancer germline variants. As a first step, information on whether the variant itself or the transcription levels of its associated gene can stratify patients based on survival should be considered. Next steps should include comparing variant frequency in cancer and non-cancer populations, and adjusting for the ancestry of the cancer and non-cancer cohorts. These steps could streamline the identification of candidate germline variants associated with a specific cancer type, and which should be selected for further validation

appropriate ethnic backgrounds (Fig. 5). The magnitude of this problem was recently highlighted in a review article, which reported that $78 \%$ of people recruited in genomic studies is of European ancestry [33]. These are traditional concepts in the field of genetic association studies that will have to be incorporated more thoroughly into cancer genomic studies. This need is made even more urgent because of the recent emphasis on research that aims to identify germline predisposing events in cancer patients.

\section{Conclusions}

We found high-frequency deletions upstream of the NEGR1 locus in pHGG and non-cancer cohorts. Low NEGR1 expression may be correlated with worse prognosis for pHGG patients. Our data underscore the need for efforts to identify new cancer-predisposing germline genetic events to use control populations that have been appropriately stratified based on ancestry.

\section{Supplementary information}

Supplementary information accompanies this paper at https://doi.org/10. 1186/s12881-020-01033-X.

Additional file 1 : Supplemental Figure S1. UCSC Genome Browser view of the NEGR1 locus. Layered ChIP-seq tracks for the active enhancer histone mark H3K27Ac, DNase clusters (corresponding to accessible chromatin) and transcription ( $T \times n)$ factor binding data from the ENCODE project are shown. The data indicate that the deleted region upstream of NEGR1 in PHGG patients may harbor regulatory regions. Supplemental Figure S2. The deletion upstream of NEGR1 appears to have specific effects on the transcription of this gene. (A-B) Transcription of ZRANB2, the gene immediately downstream of NEGR1, is detected in our sCRNA-seq datasets. (C) Single-cell transcriptomics data for 14,963 cells isolated from human hippocampus and cortex. This tSNE plot describes the clustering of different cell populations present in these brain regions. (D) Transcription of NEGR1 is detected in single-cells isolated from human hippocampus and cortex. Supplemental Table S1. Patient and sample information.

\section{Abbreviations}

CBTTC: Children's Brain Tumor Consortium; CNV: Copy number variation; DGV: Database of Genomic Variants; GBM: Glioblastoma; pHGG: Pediatric high-grade glioma; scRNA-seq: Single-cell RNA sequencing; WGS: Wholegenome sequencing
Acknowledgments

We thank Jennifer Howe and Jeffrey MacDonald for assistance and helpful discussions.

\section{Authors' contributions}

$A B, M Z, Y Z$ and $M H$ performed the experiments and analyses described in the manuscript. DB, ACR, SWS and MG designed the experiments and supervised the trainees' work. All authors have read and approved the manuscript.

\section{Funding}

Funding for this work was provided by the Canadian Institutes of Health Research (CIHR) early career award (Institute of Cancer Research) to MG (ICT156651); a Natural Sciences and Engineering Research Council (NSERC) Discovery grant to MG; an NSERC Undergraduate Student Research Award to $A B$. Funding bodies did not play any role in the design of the study and collection, analysis, and interpretation of data and in writing the manuscript.

\section{Availability of data and materials}

WGS datasets were described in Hoffman et al. [5] and were deposited to the European Genome-phenome Archive (EGA) under accession number EGAS00001003432 (https://ega-archive.org/studies/EGAS00001003432). scRNA-seq datasets were also described in Hoffman et al. [5] and were deposited to the Gene Expression Omnibus (GEO) under accession number GSE117599. The CBTTC dataset is hosted on Kids First Data Resource Portal and can be accessed via DOl:https://doi.org/10.24370/SD_BHJXBDQK. Population genetic analysis of CNVs used publicly available data in the Database of Genomic Variants (DGV) via http://dgv.tcag.ca/dgv/app/home. Initial assessment of the CNVs tested Canadian parental controls present in the MSSNG dataset, which is an open science resource available through a Data Access Committee (see https://www.mss.ng). PGCP genome data files are publicly available at www.personalgenomes.ca

\section{Ethics approval and consent to participate}

All samples were collected and used for research with appropriate written informed consent and with approval by the Health Research Ethics Board of Alberta, the Research Ethics Board of the Hospital for Sick Children (Toronto, ON) and of the CBTTC. Written consent to participate was obtained from the parents or legal guardians of individuals who had not reached the age of majority in their jurisdiction. Written consent to participate was obtained directly from individuals legally considered adults in their jurisdictions.

\section{Consent for publication}

Not applicable.

\section{Competing interests}

SWS holds the GlaxoSmithKline-CIHR Chair in Genome Sciences at The Hospital for Sick Children and University of Toronto. SWS is on the Scientific Advisory Committees of Population Bio and Deep Genomics; intellectual property from his research held at the Hospital for Sick Children is licensed to Athena Diagnostics, and separately to Lineagen. These relationships did not influence data interpretation or presentation during this study, but are disclosed for potential future consideration. 


\section{Author details}

${ }^{1}$ Charbonneau Cancer Institute, Cumming School of Medicine, University of Calgary, Calgary, Alberta, Canada. ${ }^{2}$ Alberta Children's Hospital Research Institute, Cumming School of Medicine, University of Calgary, Calgary, Alberta, Canada. ${ }^{3}$ Department of Biological Sciences, Faculty of Science, University of Calgary, Calgary, Alberta, Canada. ${ }^{4}$ The Centre for Applied Genomics and Program in Genetics and Genome Biology, The Hospital for Sick Children Research Institute, Toronto, Ontario, Canada. ${ }^{5}$ Department of Molecular Genetics, University of Toronto, Toronto, Ontario, Canada. ${ }^{6}$ McLaughlin Centre, University of Toronto, Toronto, Ontario, Canada. ${ }^{7}$ The Children's Hospital of Philadelphia, Philadelphia, PA, USA. ${ }^{8}$ Department of Biochemistry and Molecular Biology, Cumming School of Medicine, University of Calgary, Calgary, Alberta, Canada. ${ }^{9}$ Departments of Oncology and Community Health Sciences, Cumming School of Medicine, University of Calgary, Calgary, Alberta, Canada.

\section{Received: 11 February 2020 Accepted: 23 April 2020} Published online: 06 May 2020

\section{References}

1. Curtin S, Minino A, Anderson R. Declines in cancer death rates among children and adolescents in the United States, 1999-2014. NCHS data brief, no 257; 2016

2. Ostrom QT, Gittleman H, Fulop J, Liu M, Blanda R, Kromer C, Wolinsky Y, CBSJ K. CBTRUS statistical report: Primary brain and central nervous system tumors diagnosed in the United States in 2006-2010. Neuro Oncol. 2015; 17(4 suppl):iv1-iv62.

3. Cohen KJ, Pollack IF, Zhou T, Buxton A, Holmes EJ, Burger PC, et al, Temozolomide in the treatment of high-grade gliomas in children: a report from the Children's oncology group. Neuro-Oncology. 2011;13:317-23.

4. Chiang KL, Chang KP, Lee YY, Huang PI, Hsu TR, Chen YW, et al. Role of temozolomide in the treatment of newly diagnosed diffuse brainstem glioma in children: experience at a single institution. Childs Nerv Syst. 2010; 26:1035-41.

5. Hoffman M, Gillmor AH, Kunz DJ, Johnston MJ, Nikolic A, Narta K, et al. Intratumoral genetic and functional heterogeneity in pediatric glioblastoma. Cancer Res. 2019:79:2111-23.

6. Schwartzentruber J, Korshunov A, Liu XY, Jones DTW, Pfaff E, Jacob K, et al. Driver mutations in histone $\mathrm{H} 3.3$ and chromatin remodelling genes in paediatric glioblastoma. Nature. 2012;482:226-31..

7. Salloum R, McConechy MK, Mikael LG, Fuller C, Drissi R, DeWire M, et al. Characterizing temporal genomic heterogeneity in pediatric high-grade gliomas. Acta Neuropathol Commun. 2017;5:78.

8. Vinci M, Burford A, Molinari V, Kessler K, Popov S, Clarke M, et al. Functional diversity and cooperativity between subclonal populations of pediatric glioblastoma and diffuse intrinsic pontine glioma cells. Nat Med. 2018;24: 1204-15.

9. Brennan CW, Verhaak RGW, McKenna A, Campos B, Noushmehr H, Salama SR, et al. The somatic genomic landscape of glioblastoma. Cell. 2013;155: 462-77.

10. Mackay A, Burford A, Molinari V, Jones DTW, Izquierdo E, Brouwer-Visser J, et al. Molecular, pathological, radiological, and immune profiling of nonbrainstem pediatric high-grade Glioma from the HERBY phase II randomized trial. Cancer Cell. 2018;33:829-42.

11. Mackay A, Burford A, Carvalho D, Izquierdo E, Fazal-Salom J, Taylor KR, et al. Integrated molecular meta-analysis of 1,000 pediatric high-grade and diffuse intrinsic Pontine Glioma. Cancer Cell. 2017;32:520-37.

12. Sturm D, Witt H, Hovestadt $V$, Khuong-Quang DA, Jones DTW, Konermann $\mathrm{C}$, et al. Hotspot mutations in $\mathrm{H} 3 \mathrm{~F} 3 \mathrm{~A}$ and IDH1 define distinct epigenetic and biological subgroups of Glioblastoma. Cancer Cell. 2012;22:425-37.

13. Khuong-Quang DA, Buczkowicz P, Rakopoulos P, Liu XY, Fontebasso AM, Bouffet $E$, et al. K27M mutation in histone H3.3 defines clinically and biologically distinct subgroups of pediatric diffuse intrinsic pontine gliomas. Acta Neuropathol. 2012;124:439-47.

14. Larson JD, Kasper LH, Paugh BS, Jin H, Wu G, Kwon CH, et al. Histone H3.3 K27M Accelerates Spontaneous Brainstem Glioma and Drives Restricted Changes in Bivalent Gene Expression. Cancer Cell. 2019;35:140-55.

15. Pathania M, De Jay N, Maestro N, Harutyunyan AS, Nitarska J, Pahlavan P et al. H3.3 K27M cooperates with Trp53 loss and PDGFRA gain in mouse embryonic neural progenitor cells to induce invasive high-grade Gliomas. Cancer Cell. 2017;32:684-700.
16. Gröbner SN, Worst BC, Weischenfeldt J, Buchhalter I, Kleinheinz K, Rudneva VA, et al. The landscape of genomic alterations across childhood cancers. Nature. 2018;555:321-7.

17. Yuen RKC, Merico D, Bookman M, Howe JL, Thiruvahindrapuram B, Patel RV et al. Whole genome sequencing resource identifies 18 new candidate genes for autism spectrum disorder. Nat Neurosci. 2017;20:602-11.

18. Reuter MS, Walker S, Thiruvahindrapuram B, Whitney J, Cohn I, Sondheimer N, et al. The personal genome project Canada: findings from whole genome sequences of the inaugural 56 participants. CMAJ. 2018;190:E126-36.

19. MacDonald JR, Ziman R, Yuen RKC, Feuk L, Scherer SW. The database of genomic variants: a curated collection of structural variation in the human genome. Nucleic Acids Res. 2014;42:D986-92.

20. Habib N, Avraham-Davidi I, Basu A, Burks T, Shekhar K, Hofree M, et al. Massively parallel single-nucleus RNA-seq with DroNc-seq. Nat Methods. 2017;14:955-8.

21. Paugh BS, Qu C, Jones C, Liu Z, Adamowicz-Brice M, Zhang J, et al. Integrated molecular genetic profiling of pediatric high-grade gliomas reveals key differences with the adult disease. J Clin Oncol. 2010;28:3061-8.

22. Kim H, Hwang J-S, Lee B, Hong J, Lee S. Newly identified Cancer-associated role of human neuronal growth regulator 1 (NEGR1). J Cancer. 2014:5:598-608.

23. Szczurkowska J, Pischedda F, Pinto B, Manago F, Haas CA, Summa M, et al. NEGR1 and FGFR2 cooperatively regulate cortical development and core behaviours related to autism disorders in mice. Brain. 2018;141:2772-94.

24. Singh K, Loreth D, Pöttker B, Hefti K, Innos J, Schwald K, et al. Neuronal growth and behavioral alterations in mice deficient for the psychiatric disease-associated negr1 gene. Front Mol Neurosci. 2018;11:1-14.

25. Takita J, Chen Y, Okubo J, Sanada M, Adachi M, Ohki K, et al. Aberrations of NEGR1 on 1p31 and MYEOV on 11q13 in neuroblastoma. Cancer Sci. 2011; 102:1645-50.

26. Ijaz H, Koptyra M, Gaonkar KS, Rokita JL, Baubet VP, Tauhid L, et al. Pediatric High Grade Glioma Resources From the Children's Brain Tumor Tissue Consortium (CBTTC) and Pediatric Brain Tumor Atlas (PBTA). bioRxiv. 2019. https://doi.org/10.1101/656587.

27. Brahmer JR, Drake CG, Wollner I, Powderly JD, Picus J, Sharfman WH, et al. Phase I study of single-agent anti-programmed death-1 (MDX-1106) in refractory solid tumors: safety, clinical activity, pharmacodynamics, and immunologic correlates. J Clin Oncol. 2010;28:3167-75.

28. Sznol M, Chen L. Antagonist antibodies to PD-1 and B7-H1 (PD-L1) in the treatment of advanced human cancer. Clin Cancer Res. 2013;19:1021-34.

29. Chapoval Al, Smithson G, Brunick L, Mesri M, Boldog FL, Andrew D, et al. BTNL8, a butyrophilin-like molecule that costimulates the primary immune response. Mol Immunol. 2013;56:819-28.

30. Lebrero-Fern Andez C, Wenzel UA, Akeus P, Wang Y, Strid H, Simr En M, et al. Altered expression of Butyrophilin (BTN) and BTN-like (BTNL) genes in intestinal inflammation and colon cancer; altered expression of Butyrophilin (BTN) and BTN-like (BTNL) genes in intestinal inflammation and colon cancer. Inflamm Dis. 2016;4:191-200.

31. Zarrei M, MacDonald JR, Merico D, Scherer SW. A copy number variation map of the human genome. Nat Rev Genet. 2015;16:172-83.

32. Zhang J, Walsh MF, Wu G, Edmonson MN, Gruber TA, Easton J, et al. Germline mutations in predisposition genes in pediatric cancer. N Engl J Med. 2015;373:2336-46.

33. Sirugo G, Williams SM, Tishkoff SA. The missing diversity in human genetic studies. Cell. 2019;177:26-31.

\section{Publisher's Note}

Springer Nature remains neutral with regard to jurisdictional claims in published maps and institutional affiliations. 